\title{
Mappings on some reflexive algebras characterized by action on zero products or Jordan zero products
}

\author{
Yunhe Chen and Jiankui Li * \\ Department of Mathematics, East China University of Science and Technology \\ Shanghai 200237, P. R. China
}

\begin{abstract}
Let $\mathcal{L}$ be a subspace lattice on a Banach space $X$ and let $\delta: \operatorname{Alg} \mathcal{L} \rightarrow B(X)$ be a linear mapping. If $\vee\left\{L \in \mathcal{L}: L_{-} \nsupseteq L\right\}=X$ or $\wedge\left\{L_{-}: L \in \mathcal{L}, L_{-} \nsupseteq L\right\}=(0)$, we show that the following three conditions are equivalent: (1) $\delta(A B)=\delta(A) B+A \delta(B)$ whenever $A B=0$; (2) $\delta(A B+B A)=\delta(A) B+A \delta(B)+\delta(B) A+B \delta(A)$ whenever $A B+B A=0 ;(3) \delta$ is a generalized derivation and $\delta(I) \in(\operatorname{Alg} \mathcal{L})^{\prime}$. If $\vee\left\{L \in \mathcal{L}: L_{-} \nsupseteq L\right\}=X$ or $\wedge\left\{L_{-}: L \in \mathcal{L}, L_{-} \nsupseteq L\right\}=(0)$ and $\delta$ satisfies $\delta(A B+B A)=\delta(A) B+A \delta(B)+\delta(B) A+B \delta(A)$ whenever $A B=0$, we obtain that $\delta$ is a generalized derivation and $\delta(I) A \in(\operatorname{Alg} \mathcal{L})^{\prime}$ for every $A \in \operatorname{Alg} \mathcal{L}$. We also prove that if $\vee\left\{L \in \mathcal{L}: L_{-} \nsupseteq L\right\}=X$ and $\wedge\left\{L_{-}: L \in \mathcal{L}, L_{-} \nsupseteq L\right\}=(0)$, then $\delta$ is a local generalized derivation if and only if $\delta$ is a generalized derivation.
\end{abstract}

Keywords: Derivation, Jordan derivation, Reflexive algebra

Mathematics Subject Classification(2000). 47L35, 17B40

\section{Introduction}

Throughout this paper, let $X$ be a Banach space over the real or complex field $\mathbb{F}$ and $X^{*}$ be the topological dual of $X$. When $X$ is a Hilbert space, we change it to $H$. We denote by $B(X)$ the set of all bounded linear operators on $X$. For $A \in B(X)$, we denote by $A^{*}$ the adjoint of $A$. A subspace of $X$ means a norm closed linear manifold. For a subset $L \subseteq X$, denote by $L^{\perp}$ the annihilator of $L$, that is, $L^{\perp}=\left\{f \in X^{*}: f(x)=0\right.$ for all $\left.x \in L\right\}$. By a subspace lattice on $X$, we mean a collection $\mathcal{L}$ of subspaces of $X$ with $(0)$ and $X$ in $\mathcal{L}$ such that for every family $\left\{M_{r}\right\}$ of elements of $\mathcal{L}$, both $\wedge M_{r}$ and $\vee M_{r}$ belong to $\mathcal{L}$, where $\wedge M_{r}$ denotes the intersection of $\left\{M_{r}\right\}$ and $\vee M_{r}$ denotes the closed linear span of $\left\{M_{r}\right\}$. We use $\operatorname{Alg} \mathcal{L}$ to denote the algebra of operators in $B(X)$ that leave members of $\mathcal{L}$ invariant.

${ }^{*}$ Corresponding author. E-mail address:jiankuili@yahoo.com 
Let $x \in X$ and $f \in X^{*}$ be non-zero. The rank-one operator $x \otimes f$ is defined by $y \mapsto f(y) x$ for $y \in X$. If $\mathcal{L}$ is a subspace lattice on $X$ and $E \in \mathcal{L}$, we define

$$
E_{-}=\vee\{F \in \mathcal{L}: F \nsupseteq E\}, E_{+}=\wedge\{F \in \mathcal{L}: F \nsubseteq E\}
$$

and

$$
\mathcal{J}_{\mathcal{L}}=\left\{L \in \mathcal{L}: L \neq(0) \text { and } L_{-} \neq X\right\}, \mathcal{P}_{\mathcal{L}}=\left\{L \in \mathcal{L}: L_{-} \nsupseteq L\right\} .
$$

It is obvious that $\mathcal{P}_{\mathcal{L}} \subseteq \mathcal{J}_{\mathcal{L}}$. It is well known that a rank one operator $x \otimes f \in \operatorname{Alg} \mathcal{L}$ if and only if there exists a $K \in \mathcal{J}_{\mathcal{L}}$ such that $x \in K$ and $f \in K_{-}^{\perp}$. A subspace lattice $\mathcal{L}$ is called a completely distributive lattice if $L=\vee\left\{E \in \mathcal{L}: E_{-} \nsupseteq L\right\}$ for every $L \in \mathcal{L}$ (see 14]); $\mathcal{L}$ is called a $\mathcal{J}$-subspace lattice if $L \wedge L_{-}=(0)$ for every $L \in \mathcal{J}_{\mathcal{L}}, X=\vee\left\{L: L \in \mathcal{J}_{\mathcal{L}}\right\}$ and $\wedge\left\{L_{-}: L \in \mathcal{J}_{\mathcal{L}}\right\}=(0)$ (see [15). A totally ordered subspace lattice $\mathcal{N}$ is called a nest. Recall that $\mathcal{N}$ is a discrete nest if a nest $\mathcal{N}$ satisfies $N_{-} \neq N$ for every non-trivial subspace $N$ in $\mathcal{N}$.

We say that $\mathcal{L}$ is a $\mathcal{P}$-subspace lattice on $X$ if $\vee\left\{L: L \in \mathcal{P}_{\mathcal{L}}\right\}=X$ or $\wedge\left\{L L_{-}: L \in \mathcal{P}_{\mathcal{L}}\right\}=(0)$. It is obvious that this class of subspace lattices contains $\mathcal{J}$-subspace lattices, discrete nests and subspace lattices with $X_{-} \neq X$ or $(0)_{+} \neq(0)$. The following example is also a $\mathcal{P}$-subspace lattice.

Example 1.1. Let $\left\{e_{n}: n \in \mathbb{N}\right\}$ be an orthonormal basis of $H, P_{n}=\operatorname{span}\left\{e_{i}: i=1, \ldots, n\right\}, \xi=$ $\sum_{n=1}^{\infty} \frac{1}{n} e_{n}$ and $P_{\xi}$ be the orthogonal projection from $H$ onto the one-dimensional subspace of $H$ generated by $\xi$. It follows from [20, Theorem 2.11] and [7, Lemma 3.2] that $\mathcal{L}=\left\{0, I, P_{n}, P_{\xi}, P_{\xi} \vee\right.$ $\left.P_{n}: n=1,2, \cdots\right\}$ is a reflexive $\mathcal{P}$-subspace lattice.

In a Hilbert space, we disregard the distinction between a closed subspace and the orthogonal projection onto it. A subspace lattice on a Hilbert space $H$ is called a commutative subspace lattice (or CSL for short) if it consists of mutually commuting projections. In the paper, we assume that $H$ is a complex separable Hilbert space.

Let $\delta$ be a linear mapping from a unital algebra $\mathcal{A}$ into an $\mathcal{A}$-bimodule $\mathcal{M}$. Recall that $\delta$ is a derivation (respectively generalized derivation) if $\delta(A B)=\delta(A) B+A \delta(B)$ (respectively $\delta(A B)=\delta(A) B+A \delta(B)-A \delta(I) B)$ for all $A, B$ in $\mathcal{A}$. We say that $\delta$ is derivable at $Z \in \mathcal{A}$ if $\delta(A B)=\delta(A) B+A \delta(B)$ for any $A, B \in \mathcal{A}$ with $A B=Z ; \delta$ is Jordan derivable at $Z \in \mathcal{A}$ if $\delta(A B+B A)=\delta(A) B+A \delta(B)+\delta(B) A+B \delta(A)$ for any $A, B \in \mathcal{A}$ with $A B+B A=Z$. If $\delta(A B+B A)=\delta(A) B+A \delta(B)+\delta(B) A+B \delta(A)$ for any $A, B \in \mathcal{A}$ with $A B=0$, we say that $\delta$ has $W J D$ (weak Jordan derivation) property.

In recent years, there have been a number of papers on the study of conditions under which derivations and Jordan derivations of operator algebras can be completely determined by the action on some subsets of operator algebras (for example, see [1, 3, 8, 10, 21]). For instance, Zhao and Zhu in 21] showed that every linear mapping $\delta$ from a triangular algebra $\mathcal{T}$ into itself satisfying WJD property is a derivation. In [8], Jiao and Hou proved that every additive mapping $\delta$ derivable or Jordan derivable at zero point on some nest algebras has the form $\delta(A)=\tau(A)+c A$ for some additive derivation $\tau$ and some scalar $c \in \mathbb{F}$.

The purpose of this paper is to consider some mappings which behave like derivations on $\mathcal{P}$-subspace lattice algebras and completely distributive commutative subspace lattice (CDCSL) algebras.

In Section 2, we show that every linear (respectively bounded linear) mapping $\delta$ on $\mathcal{P}$ subspace lattice (respectively CDCSL) algebras Jordan derivable at zero point is a generalized derivation and $\delta(I) \in(\operatorname{Alg} \mathcal{L})^{\prime}$. 
In Section 3, for a $\mathcal{P}$-subspace lattice algebra $\operatorname{Alg} \mathcal{L}$, we obtain that $\delta$ satisfies WJD property if and only if $\delta$ is a generalized derivation and $\delta(I) A \in(\operatorname{Alg} \mathcal{L})^{\prime}$ for every $A \in \operatorname{Alg} \mathcal{L}$.

In Section 4, we investigate derivable mappings at zero point and some linear mappings which behave like left (respectively right) multipliers, isomorphisms or local generalized derivations on $\mathcal{P}$-subspace lattice algebras. One of the main results of the section is that if $\vee\left\{L \in \mathcal{L}: L_{-} \nsupseteq\right.$ $L\}=X$ and $\wedge\left\{L_{-}: L \in \mathcal{L}, L_{-} \nsupseteq L\right\}=(0)$, then $\delta$ is a local generalized derivation from $\operatorname{Alg} \mathcal{L}$ into $B(X)$ if and only if $\delta$ is a generalized derivation.

The following proposition will be used in our proofs.

Proposition 1.2 ([19, Proposition 1.1]). Let $E$ and $F$ be non-zero subspaces of $X$ and $X^{*}$, respectively. Let $\Phi: E \times F \rightarrow B(X)$ be a bilinear mapping such that $\Phi(x, f) k e r(f) \subseteq \mathbb{F} x$ for all $x \in E$ and $f \in F$. Then there exist two linear mappings $T: E \rightarrow X$ and $S: F \rightarrow X^{*}$ such that $\Phi(x, f)=T x \otimes f+x \otimes S f$ for all $x \in E$ and $f \in F$.

\section{Jordan derivable Mappings at zero point}

The following lemma is included in the proof of [8, Theorem 3.1]. We leave the proof to readers.

Lemma 2.1. If $\delta$ is Jordan derivable at zero point from a unital algebra $\mathcal{A}$ into its unital bimodule, then for any idempotents $P$ and $Q$ in $\mathcal{A}$, the following hold:

(1) $\delta(I) P=P \delta(I)$;

(2) $\delta(P)=\delta(P) P+P \delta(P)-P \delta(I)$;

(3) $\delta(P Q+Q P)=\delta(P) Q+P \delta(Q)+\delta(Q) P+Q \delta(P)-\delta(I)(P Q+Q P)$.

For a subspace lattice $\mathcal{L}$ and a subspace $E \in \mathcal{P}_{\mathcal{L}}$, we denote by $\mathcal{T}_{E}$ the ideal $\operatorname{span}\{x \otimes f: x \in$ $\left.E, f \in E_{-}^{\perp}\right\}$ of $\operatorname{Alg} \mathcal{L}$.

Lemma 2.2. If $\mathcal{L}$ is a subspace lattice on $X$ and $E$ is in $\mathcal{P}_{\mathcal{L}}$, then for every $x$ in $E$ and every $f$ in $E_{-}^{\perp}, x \otimes f$ is a linear combination of idempotents in $\mathcal{T}_{E}$.

Proof. Suppose $f(x) \neq 0$, then $x \otimes f=f(x)\left(\frac{1}{f(x)} x \otimes f\right)$, where $\frac{1}{f(x)} x \otimes f$ is an idempotent in $\mathcal{T}_{E}$. Suppose $f(x)=0$. Since $E \in \mathcal{P}_{\mathcal{L}}$, there exist $z \in E$ and $g \in E_{-}^{\perp}$ such that $g(z)=1$.

Case 1. If $g(x)=\mu \neq 0$, then $x \otimes f=x \otimes\left(\frac{1}{\mu} g+f\right)-x \otimes \frac{1}{\mu} g$, where $x \otimes\left(\frac{1}{\mu} g+f\right)$ and $x \otimes \frac{1}{\mu} g$ are idempotents in $\mathcal{T}_{E}$.

Case 2. If $f(z)=\lambda \neq 0$, then $x \otimes f=\left(x+\frac{1}{\lambda} z\right) \otimes f-\frac{1}{\lambda} z \otimes f$, where $\left(x+\frac{1}{\lambda} z\right) \otimes f$ and $\frac{1}{\lambda} z \otimes f$ are idempotents in $\mathcal{T}_{E}$.

Case 3. If $f(z)=g(x)=0$, then $x \otimes f=\frac{1}{4}((z+x) \otimes(g+f)+(z-x) \otimes(g-f)-(z+x) \otimes$ $(g-f)-(z-x) \otimes(g+f))$, where $(z+x) \otimes(g+f),(z-x) \otimes(g-f),(z+x) \otimes(g-f)$ and $(z-x) \otimes(g+f)$ are idempotents in $\mathcal{T}_{E}$. The proof is complete.

Lemma 2.3. Let $\mathcal{L}$ be a subspace lattice on $X, E$ be in $P_{\mathcal{L}}$ and $\delta$ be a linear mapping from $\operatorname{Alg} \mathcal{L}$ into $B(X)$. If $\delta$ is Jordan derivable at zero point, then for every idempotent $P$ in $\mathcal{T}_{E}$ and every $A$ in $\operatorname{Alg} \mathcal{L}$, the following hold:

(1) $\delta(A P+P A)=\delta(A) P+A \delta(P)+\delta(P) A+P \delta(A)-\delta(I)(A P+P A)$;

(2) $\delta(P A P)=\delta(P) A P+P \delta(A) P+P A \delta(P)-2 \delta(I) P A P$. 
Proof. (1) For every idempotent $P \in \mathcal{T}_{E}$ and every $A \in \operatorname{Alg} \mathcal{L}$, since $P^{\perp} A P^{\perp} P+P P^{\perp} A P^{\perp}=0$, by assumption we have

$$
\delta\left(P^{\perp} A P^{\perp}\right) P+P^{\perp} A P^{\perp} \delta(P)+\delta(P) P^{\perp} A P^{\perp}+P \delta\left(P^{\perp} A P^{\perp}\right)=0 .
$$

Since $A-P^{\perp} A P^{\perp}=P A+P^{\perp} A P \in \mathcal{T}_{E}$, it follows from Lemmas 2.1 and 2.2 that

$$
\begin{aligned}
\delta(A P+P A)= & \delta\left(\left(A-P^{\perp} A P^{\perp}\right) P+P\left(A-P^{\perp} A P^{\perp}\right)\right) \\
= & \delta\left(A-P^{\perp} A P^{\perp}\right) P+\left(A-P^{\perp} A P^{\perp}\right) \delta(P)+\delta(P)\left(A-P^{\perp} A P^{\perp}\right) \\
& +P \delta\left(A-P^{\perp} A P^{\perp}\right)-\delta(I)(A P+P A) \\
= & \delta(A) P+A \delta(P)+\delta(P) A+P \delta(A)-\delta(I)(A P+P A) \\
& -\left(\delta\left(P^{\perp} A P^{\perp}\right) P+P^{\perp} A P^{\perp} \delta(P)+\delta(P) P^{\perp} A P^{\perp}+P \delta\left(P^{\perp} A P^{\perp}\right)\right) \\
= & \delta(A) P+A \delta(P)+\delta(P) A+P \delta(A)-\delta(I)(A P+P A) .
\end{aligned}
$$

(2) The substitution $A P+P A$ for $A$ in (1) gives (2).

One of the main results of this section is the following theorem.

Theorem 2.4. Let $\mathcal{L}$ be a subspace lattice on $X$ such that $\vee\left\{L: L \in \mathcal{P}_{\mathcal{L}}\right\}=X$ and $\delta$ be a linear mapping from $\operatorname{Alg} \mathcal{L}$ into $B(X)$. Then $\delta$ is Jordan derivable at zero point if and only if $\delta$ is a generalized derivation and $\delta(I) \in(\operatorname{Alg} \mathcal{L})^{\prime}$, where $(\operatorname{Alg} \mathcal{L})^{\prime}$ is the commutant of $\operatorname{Alg} \mathcal{L}$ in $B(X)$. In particular, if $\delta(I)=0$, then $\delta$ is Jordan derivable at zero point if and only if $\delta$ is a derivation.

Proof. The sufficiency is obvious, so we only need to prove the necessity. Let $E \in \mathcal{P}_{\mathcal{L}}, z \in E$ and $g \in E_{-}^{\perp}$ with $g(z)=1$. We divide the proof into several claims.

Claim 1. $\delta(I) \in(\operatorname{Alg} \mathcal{L})^{\prime}$.

For all $x \in E, f \in E_{-}^{\perp}$ and $T \in \operatorname{Alg} \mathcal{L}$, by Lemmas 2.1 and 2.2 we have $\delta(I) T x \otimes f=$ $T x \otimes f \delta(I)=T \delta(I) x \otimes f$. That is, $\delta(I) T x=T \delta(I) x$ for every $x \in E$. Since $\vee\left\{E: E \in \mathcal{P}_{\mathcal{L}}\right\}=X$, it follows that $\delta(I) \in(\operatorname{Alg} \mathcal{L})^{\prime}$.

Now define $\tau(A)=\delta(A)-\delta(I) A$ for $A \in \operatorname{Alg} \mathcal{L}$. It is easy to see that $\tau$ is Jordan derivable at zero point and $\tau(I)=0$.

Claim 2. $\tau(x \otimes f) k e r(f) \subseteq \mathbb{F} x$, for all $x \in E$ and $f \in E_{-}^{\perp}$.

Case 1. If $f(x)=\mu \neq 0$, then by Lemma 2.1, we have

$$
\left.\tau\left(\frac{1}{\mu} x \otimes f\right)\right)=\tau\left(\frac{1}{\mu} x \otimes f\right)\left(\frac{1}{\mu} x \otimes f\right)+\left(\frac{1}{\mu} x \otimes f\right) \tau\left(\frac{1}{\mu} x \otimes f\right) .
$$

Thus $\tau(x \otimes f) k e r(f) \subseteq \mathbb{F} x$.

Case 2. If $f(x)=0$ and $f(z) \neq 0$, then by Case 1 , for every $y \in k e r(f)$, we have

$$
\begin{aligned}
\tau((z+x) \otimes f) y & =\lambda_{1}(z+x), \\
\tau((z-x) \otimes f) y & =\lambda_{2}(z-x), \\
\tau(z \otimes f) y & =\lambda_{3} z,
\end{aligned}
$$

for some $\lambda_{1}, \lambda_{2}$ and $\lambda_{3} \in \mathbb{F}$. By the above equations, it follows that

$$
2 \lambda_{3} z=\left(\lambda_{1}+\lambda_{2}\right) z+\left(\lambda_{1}-\lambda_{2}\right) x
$$


and the independence of $z$ and $x$ implies $\lambda_{1}=\lambda_{2}=\lambda_{3}$. Hence

$$
\tau(x \otimes f) y=\tau((z+x) \otimes f) y-\tau(z \otimes f) y=\lambda_{1} x .
$$

This means $\tau(x \otimes f) k e r(f) \subseteq \mathbb{F} x$.

Case 3. Suppose that $f(x)=0$ and $f(z)=0$. Since $z \otimes(g+f)$ and $z \otimes(g-f)$ are idempotents in $\mathcal{T}_{E}$, it follows from Lemma 2.3 that

$$
\begin{aligned}
\tau((z \otimes(g+f))(x \otimes g)(z \otimes(g+f))) & \\
= & \tau(z \otimes(g+f))(x \otimes g)(z \otimes(g+f))+(z \otimes(g+f)) \tau(x \otimes g)(z \otimes(g+f)) \\
& +(z \otimes(g+f))(x \otimes g) \tau(z \otimes(g+f)), \\
\tau((z \otimes(g-f))(x \otimes g)(z \otimes(g-f))) & \\
= & \tau(z \otimes(g-f))(x \otimes g)(z \otimes(g-f))+(z \otimes(g-f)) \tau(x \otimes g)(z \otimes(g-f)) \\
& +(z \otimes(g-f))(x \otimes g) \tau(z \otimes(g-f)),
\end{aligned}
$$

and

$$
\begin{aligned}
& \tau((z \otimes g)(x \otimes g)(z \otimes g)) \\
& \quad=\tau(z \otimes g)(x \otimes g)(z \otimes g)+(z \otimes g) \tau(x \otimes g)(z \otimes g)+(z \otimes g)(x \otimes g) \tau(z \otimes g) .
\end{aligned}
$$

From the above three equations, we have

$$
\begin{aligned}
0 & =\tau((z \otimes f)(x \otimes g)(z \otimes f)) \\
& =\tau(z \otimes f)(x \otimes g)(z \otimes f)+(z \otimes f) \tau(x \otimes g)(z \otimes f)+(z \otimes f)(x \otimes g) \tau(z \otimes f) \\
& =\tau(z \otimes f)(x \otimes f)+(z \otimes f) \tau(x \otimes g)(z \otimes f) .
\end{aligned}
$$

Thus

$$
\tau(z \otimes f) x=-f(\tau(x \otimes g) z) z .
$$

Hence by (2.1), Lemmas 2.2 and 2.3 , it follows that

$$
\begin{aligned}
\tau(x \otimes f)= & \tau((z \otimes f)(x \otimes g)+(x \otimes g)(z \otimes f)) \\
= & -f(\tau(x \otimes g) z) z \otimes g+(z \otimes f) \tau(x \otimes g) \\
& +\tau(x \otimes g)(z \otimes f)+(x \otimes g) \tau(z \otimes f) .
\end{aligned}
$$

Let $y$ be in $\operatorname{ker}(f)$. Applying the above equations to $y$ gives

$$
\tau(x \otimes f) y=-g(y) f(\tau(x \otimes g) z) z+f(\tau(x \otimes g) y) z+g(\tau(z \otimes f) y) x .
$$

Notice that (2.2) is valid for all $z \in E$ satisfying $g(z)=1$ and $f(z)=0$. If $g(x)=\mu \neq 0$, replacing $z$ by $\frac{1}{\mu} x$ in (2.2), we have $\tau(x \otimes f) y \in \mathbb{F} x$. If $g(x)=0$, by the proof of [18, Lemma 2.3], we have $g(y) f(\tau(x \otimes g) z)-f(\tau(x \otimes g) y)=0$, whence $\tau(x \otimes f) y=g(\tau(z \otimes f) y) x \in \mathbb{F} x$.

Claim 3. $\tau$ is a derivation.

By Claim 2 and Proposition 1.2, there exist linear mappings $T: E \rightarrow X$ and $S: E_{-}^{\perp} \rightarrow X^{*}$ such that

$$
\tau(x \otimes f)=T x \otimes f+x \otimes S f,
$$


for all $x \in E$ and $f \in E_{-}^{\perp}$. It follows from Lemmas 2.2 and 2.3 that for every $A \in \operatorname{Alg} \mathcal{L}$,

$$
\tau(A x \otimes g+x \otimes g A)=\tau(A) x \otimes g+A \tau(x \otimes g)+\tau(x \otimes g) A+x \otimes g \tau(A) .
$$

By (2.3) and (2.4), we have

$$
\begin{aligned}
T A x \otimes g & +A x \otimes S g+T x \otimes A^{*} g+x \otimes S A^{*} g \\
& =\tau(A) x \otimes g+A T x \otimes g+A x \otimes S g+T x \otimes A^{*} g+x \otimes A^{*} S g+x \otimes \tau(A)^{*} g .
\end{aligned}
$$

That is,

$$
(\tau(A)+A T-T A) x \otimes g=x \otimes\left(S A^{*}-\tau(A)^{*}-A^{*} S\right) g .
$$

Thus there exists a linear mapping $\lambda: \operatorname{Alg} \mathcal{L} \rightarrow \mathbb{F}$ such that

$$
\tau(A) x=(T A-A T) x+\lambda(A) x,
$$

for all $A \in \operatorname{Alg} \mathcal{L}$ and $x \in E$. Hence by (2.5), for all $A, B$ in $\operatorname{Alg} \mathcal{L}$ and $x$ in $E$,

$$
\tau(A B) x=(\tau(A) B+A \tau(B)) x+\lambda(A B) x-\lambda(A) B x-\lambda(B) A x .
$$

In the following, we show $\lambda(A)=0$ for every $A \in \operatorname{Alg} \mathcal{L}$. Putting $A=B=z \otimes g$ and $x=z$ in (2.6) gives $\lambda(z \otimes g)=g(\tau(z \otimes g) z)$, and Lemma 2.1 (2) implies $g(\tau(z \otimes g) z)=0$. Hence

$$
\lambda(z \otimes g)=0 .
$$

Notice that (2.7) is valid for all $z$ in $E$ and $g$ in $E_{-}^{\perp}$ satisfying $g(z)=1$. Now fix $z \in E$ and $g \in E_{-}^{\perp}$ such that $g(z)=1$. Thus for all $f \in E_{-}^{\perp}$, if $f(z)=\mu \neq 0$, then $\lambda(z \otimes f)=\mu \lambda\left(z \otimes \frac{1}{\mu} f\right)=0$; if $f(z)=0$, then $\lambda(z \otimes f)=\lambda(z \otimes(g+f))-\lambda(z \otimes g)=0$. Hence $\lambda(z \otimes f)=0$ for every $f \in E_{-}^{\perp}$. Similarly, we have $\lambda(x \otimes g)=0$ for every $x \in E$. Now for every $A \in \operatorname{Alg} \mathcal{L}$, by (2.6), we have

$$
\tau(A z \otimes g) z=\tau(A) z+A \tau(z \otimes g) z-\lambda(A) z
$$

and

$$
\tau(z \otimes g A) z=\tau(z \otimes g) A z+g(\tau(A) z) z-\lambda(A) z .
$$

By Lemma 2.3 (1), we have

$$
\tau(A z \otimes g+z \otimes g A) z=\tau(A) z+A \tau(z \otimes g) z+\tau(z \otimes g) A z+g(\tau(A) z) z .
$$

Combining (2.8), (2.9) and (2.10) gives $\lambda(A)=0$ for every $A \in \operatorname{Alg} \mathcal{L}$. Then by (2.6), we obtain

$$
\tau(A B) x=(\tau(A) B+A \tau(B)) x,
$$

for all $A, B \in \operatorname{Alg} \mathcal{L}$ and $x \in E$. Since $\vee\left\{L: L \in \mathcal{P}_{\mathcal{L}}\right\}=X$, it follows that $\tau$ is a derivation. By $\delta(A)=\tau(A)+\delta(I) A$, it is easy to show that $\delta$ is a generalized derivation.

Applying the ideas in the proof of Theorem 2.4, we can obtain the following result.

Theorem 2.5. Let $\mathcal{L}$ be a subspace lattice on $X$ such that $\wedge\left\{L_{-}: L \in \mathcal{P}_{\mathcal{L}}\right\}=(0)$ and $\delta$ be a linear mapping from $\operatorname{Alg} \mathcal{L}$ into $B(X)$. Then $\delta$ is Jordan derivable at zero point if and only if $\delta$ is a generalized derivation and $\delta(I) \in(\operatorname{Alg} \mathcal{L})^{\prime}$. In particular, if $\delta(I)=0$, then $\delta$ is Jordan derivable at zero point if and only if $\delta$ is a derivation. 
Proof. We only prove the necessity. Let $x \mapsto \hat{x}$ be the canonical mapping from $X$ into $X^{* *}$, then $(x \otimes f)^{*}=f \otimes \hat{x}$ for all $x \in X$ and $f \in X^{*}$. The hypothesis $\wedge\left\{L_{-}: L \in \mathcal{P}_{\mathcal{L}}\right\}=(0)$ implies that $\vee\left\{L_{-}^{\perp}: L \in \mathcal{P}_{\mathcal{L}}\right\}=X^{*}$. With a proof similar to the proof of Theorem 2.4 we have $\delta(I) \in(\operatorname{Alg} \mathcal{L})^{\prime}$. Let $\tau(A)=\delta(A)-\delta(I) A$ for $A \in \operatorname{Alg} \mathcal{L}$. Then $\tau$ is Jordan derivable at zero point and $\tau(I)=0$. In the following, we show $\tau$ is a derivation. Let $E \in \mathcal{P}_{\mathcal{L}}$. We choose $z \in E$ and $g \in E_{-}^{\perp}$ such that $g(z)=1$. One can easily verify that for all $x \in E$ and $f \in E_{-}^{\perp}$, $\tau(x \otimes f)^{*} \operatorname{ker}(\hat{x}) \subseteq \mathbb{F} f$. Let $\Phi(f, \hat{x})=\tau(x \otimes f)^{*}$ for all $x \in E$ and $f \in E_{-}^{\perp}$. Then $\Phi$ is a bilinear mapping from $E_{-}^{\perp} \times \hat{E}$ into $B\left(X^{*}\right)$, where $\hat{E}=\{\hat{x}: x \in E\}$. Hence there exist linear mappings $T: E_{-}^{\perp} \rightarrow X^{*}$ and $S: \hat{E} \rightarrow X^{* *}$ such that

$$
\tau(x \otimes f)^{*}=\Phi(f, \hat{x})=T f \otimes \hat{x}+f \otimes S \hat{x},
$$

for all $x \in E$ and $f \in E_{-}^{\perp}$. Hence for $A \in \operatorname{Alg} \mathcal{L}$ and $f \in E_{-}^{\perp}$, we have that

$$
\left(\tau(A)^{*}+A^{*} T-T A^{*}\right) f \otimes \hat{z}=f \otimes\left(S \widehat{A z}-\widehat{\delta(A) z}-A^{* *} S \hat{z}\right) .
$$

It follows that $\tau(A)^{*} f=\left(T A^{*}-A^{*} T\right) f+\lambda(A) f$, where $\lambda: \operatorname{Alg} \mathcal{L} \rightarrow \mathbb{F}$ is a linear mapping. Hence for all $A, B \in \operatorname{Alg} \mathcal{L}$ and $f \in E_{-}^{\perp}$,

$$
\tau(A B)^{*} f=\left(B^{*} \tau(A)^{*}+\tau(B)^{*} A^{*}\right) f-\lambda(A) B^{*} f-\lambda(B) A^{*} f+\lambda(A B) f .
$$

With a proof similar to the proof of Theorem 2.4, we can prove that $\lambda(A)=0$ for every $A \in \operatorname{Alg} \mathcal{L}$. Since $\vee\left\{L_{-}^{\perp}: L \in \mathcal{P}_{\mathcal{L}}\right\}=X^{*}$, it follows that $\tau$ is a derivation. Hence $\delta$ is a generalized derivation.

Next we investigate the bounded linear mappings which are Jordan derivable at zero point on CDCSL algebras. Recall that a CSL algebra $\operatorname{Alg} \mathcal{L}$ is irreducible if and only if $(\operatorname{Alg} \mathcal{L})^{\prime}=\mathbb{C} I$, which is equivalent to the condition that $\mathcal{L} \cap \mathcal{L}^{\perp}=\{0, I\}$, where $\mathcal{L}^{\perp}=\left\{E^{\perp}: E \in \mathcal{L}\right\}$.

Lemma 2.6 (5). Let $\operatorname{Alg} \mathcal{L}$ be a CDCSL algebra on $H$. Then there exists a countable set $\left\{P_{n}: n \in \Lambda\right\}$ of mutually orthogonal projections in $\mathcal{L} \cap \mathcal{L}^{\perp}$ such that $\vee_{n} P_{n}=I$ and each $(\operatorname{Alg} \mathcal{L}) P_{n}$ is an irreducible CDCSL algebra on $P_{n} H$; moreover, $\operatorname{Alg} \mathcal{L}$ can be written as a direct sum $\operatorname{Alg} \mathcal{L}=\sum_{n} \bigoplus(\operatorname{Alg} \mathcal{L}) P_{n}$.

Lemma 2.7 ([16]). Let $\operatorname{Alg} \mathcal{L}$ be a non-trivially irreducible CDCSL algebra on $H$. Then there exists a non-trivial projection $P$ in $\mathcal{L}$ such that $P(\operatorname{Alg} \mathcal{L}) P^{\perp}$ is faithful, that is, for $T, S \in \operatorname{Alg} \mathcal{L}$, $T P(\operatorname{Alg} \mathcal{L}) P^{\perp}=\{0\}$ implies $T P=0$ and $P(\operatorname{Alg} \mathcal{L}) P^{\perp} S=\{0\}$ implies $P^{\perp} S=0$.

Lemma 2.8. Let $\operatorname{Alg} \mathcal{L}$ be an irreducible CDCSL algebra on $H$ and let $\delta: \operatorname{Alg} \mathcal{L} \rightarrow \operatorname{Alg} \mathcal{L}$ be a bounded linear mapping and $\delta(I)=0$. If $\delta$ is Jordan derivable at zero point, then $\delta$ is a derivation.

Proof. Suppose that $\mathcal{L}$ is trivial, then $\operatorname{Alg} \mathcal{L}=B(H)$ is a von Neumann algebra. It follows from [1, Theorem 3.2] that $\delta$ is a Jordan derivation. Since every von Neumann algebra is a semiprime ring, by [2, Theorem 1], $\delta$ is a derivation.

Suppose that $\mathcal{L}$ is non-trivial. Let $P$ be the non-trivial projection in $\mathcal{L}$ provided by Lemma 2.7. Since $P(\operatorname{Alg} \mathcal{L}) P^{\perp}$ is faithful, by [1, Theorem 2.1], $\delta$ is a Jordan derivation. Since every Jordan derivation on a CSL algebra is a derivation [17, Theorem 3.2], it follows that $\delta$ is a derivation. 
Theorem 2.9. Let $\operatorname{Alg} \mathcal{L}$ be a CDCSL algebra on $H$ and $\delta$ be a bounded linear mapping from $\operatorname{Alg} \mathcal{L}$ into itself. Then $\delta$ is Jordan derivable at zero point if and only if $\delta$ is a generalized derivation and $\delta(I) \in(\operatorname{Alg} \mathcal{L})^{\prime}$. In particular, if $\delta(I)=0$, then $\delta$ is Jordan derivable at zero point if and only if $\delta$ is a derivation.

Proof. We only prove the necessity. Since every rank one operator in $\operatorname{Alg} \mathcal{L}$ is a linear combination of idempotents in $\operatorname{Alg} \mathcal{L}$ [6, Lemma 2.3] and the rank one subalgebra of $\operatorname{Alg} \mathcal{L}$ is dense in $\operatorname{Alg} \mathcal{L}$ in the weak topology [9, Theorem 3], by Lemma 2.1(1), we have $\delta(I) \in(\operatorname{Alg} \mathcal{L})^{\prime}$. Let $\tau(A)=\delta(A)-\delta(I) A$ for $A \in \operatorname{Alg} \mathcal{L}$. Then $\tau$ is Jordan derivable at zero point and $\tau(I)=0$.

Let $\operatorname{Alg} \mathcal{L}=\sum_{n} \bigoplus(\operatorname{Alg} \mathcal{L}) P_{n}$ be the irreducible decomposition of $\operatorname{Alg} \mathcal{L}$ as in Lemma 2.6. Let $A$ be in $\operatorname{Alg} \mathcal{L}$ and fix an index $n$. Since $P_{n} A P_{n} P_{n}^{\perp}+P_{n}^{\perp} P_{n} A P_{n}=0$, we have

$$
\begin{aligned}
0= & \tau\left(P_{n} A P_{n} P_{n}^{\perp}+P_{n}^{\perp} P_{n} A P_{n}\right) \\
& =\tau\left(P_{n} A P_{n}\right) P_{n}^{\perp}+P_{n} A P_{n} \tau\left(P_{n}^{\perp}\right)+\tau\left(P_{n}^{\perp}\right) P_{n} A P_{n}+P_{n}^{\perp} \tau\left(P_{n} A P_{n}\right),
\end{aligned}
$$

which yields that $P_{n}^{\perp} \tau\left(P_{n} A P_{n}\right) P_{n}^{\perp}=0$. Since $P_{n} \in \mathcal{L} \cap \mathcal{L}^{\perp}$, there holds $\tau\left(A P_{n}\right)=\tau\left(A P_{n}\right) P_{n}$. By the same way, we obtain $\tau\left(A P_{n}^{\perp}\right)=\tau\left(A P_{n}^{\perp}\right) P_{n}^{\perp}$. Since

$$
0=\tau(I)=\tau\left(P_{n}+P_{n}^{\perp}\right)=\tau\left(P_{n}\right) P_{n}+\tau\left(P_{n}^{\perp}\right) P_{n}^{\perp},
$$

it follows that $\tau\left(P_{n}\right)=0$. Now define a linear mapping $\tau_{n}:(\operatorname{Alg} \mathcal{L}) P_{n} \rightarrow(\operatorname{Alg} \mathcal{L}) P_{n}$ by

$$
\tau_{n}\left(A P_{n}\right)=\tau\left(A P_{n}\right) P_{n},
$$

for every $A \in \operatorname{Alg} \mathcal{L}$. It is easy to show that $\tau_{n}$ is bounded and Jordan derivable at zero point. Since $(\operatorname{Alg} \mathcal{L}) P_{n}$ is irreducible and $\tau_{n}\left(P_{n}\right)=\tau\left(P_{n}\right) P_{n}=0$, by Lemma 2.8, $\tau_{n}$ is a derivation. Hence by $\tau(A) P_{n}=\tau\left(A P_{n}\right) P_{n}+\tau\left(A P_{n}^{\perp}\right) P_{n}=\tau_{n}\left(A P_{n}\right)$, we have $\tau$ is a derivation. Thus $\delta$ is a generalized derivation.

\section{Mappings satisfying WJD property}

Our first result in this section says that the set of all Jordan derivable mapping at zero point from a $\mathcal{P}$-subspace lattice algebra into $B(X)$ is bigger than the set of all mappings satisfying WJD property. The following lemma is included in the proof of [4, Lemma 2.6].

Lemma 3.1. If $\delta$ is a linear mapping satisfying WJD property from a unital algebra $\mathcal{A}$ into its unital bimodule, then for every idempotent $P \in \mathcal{A}$ and every $A \in \mathcal{A}$, the following hold:

(1) $\delta(I) P=P \delta(I)$ and $\delta(P)=\delta(P) P+P \delta(P)-\delta(I) P$;

(2) $\delta(P A+A P)=\delta(P) A+P \delta(A)+\delta(A) P+A \delta(P)-\delta(I) P A-P A \delta(I)$

(3) $\delta(P A+A P)=\delta(P) A+P \delta(A)+\delta(A) P+A \delta(P)-\delta(I) A P-A P \delta(I)$;

(4) $2 \delta(P A P)=2 \delta(P) A P+2 P \delta(A) P+2 P A \delta(P)-P A \delta(I)-2 \delta(I) A P-A P \delta(I)$.

Theorem 3.2. Let $\mathcal{L}$ be a subspace lattice on $X$ such that $\vee\left\{L: L \in \mathcal{P}_{\mathcal{L}}\right\}=X$ and $\delta$ be a linear mapping from $\operatorname{Alg} \mathcal{L}$ into $B(X)$. Then $\delta$ satisfies WJD property if and only if $\delta$ is a generalized derivation and $\delta(I) A \in(\operatorname{Alg} \mathcal{L})^{\prime}$ for every $A \in \operatorname{Alg} \mathcal{L}$. In particular, if $\delta(I)=0$, then $\delta$ satisfies $W J D$ property if and only if $\delta$ is a derivation. 
Proof. Since the sufficiency is evident, we will just show the necessity. Suppose $\delta$ satisfies WJD property. We claim that $\delta(I) A \in(\operatorname{Alg} \mathcal{L})^{\prime}$ for every $A \in \operatorname{Alg} \mathcal{L}$. By Lemma 3.1 (1) and the proof of Claim 1 in Theorem 2.4 we have $\delta(I) \in(\operatorname{Alg} \mathcal{L})^{\prime}$. Hence by Lemma 3.1 (2) and (3), we have that $\delta(I) A P=P A \delta(I)$ for every idempotent $P \in \operatorname{Alg} \mathcal{L}$ and every $A \in \operatorname{Alg} \mathcal{L}$. Hence for all $x \in E, f \in E_{\perp}^{\perp}$ and $T \in \operatorname{Alg} \mathcal{L}$, we have $\delta(I) A T x \otimes f=T x \otimes f A \delta(I)=T \delta(I) A x \otimes f$. Since $\vee\{L:$ $\left.L \in \mathcal{P}_{\mathcal{L}}\right\}=X$, it follows that $\delta(I) A \in(\operatorname{Alg} \mathcal{L})^{\prime}$ for every $A \in \operatorname{Alg} \mathcal{L}$. Let $\tau(A)=\delta(A)-\delta(I) A$ for $A \in \operatorname{Alg} \mathcal{L}$. It is easy to show that $\tau$ satisfies WJD property and $\tau(I)=0$. Similar to the proof of Theorem 2.4 we may show $\tau$ is a derivation and then $\delta$ is a generalized derivation.

Similarly, we have the following theorem.

Theorem 3.3. Let $\mathcal{L}$ be a subspace lattice on $X$ such that $\wedge\left\{L_{-}: L \in \mathcal{P}_{\mathcal{L}}\right\}=(0)$ and $\delta$ be a linear mapping from $\operatorname{Alg} \mathcal{L}$ into $B(X)$. Then $\delta$ satisfies WJD property if and only if $\delta$ is a generalized derivation and $\delta(I) A \in(\operatorname{Alg} \mathcal{L})^{\prime}$ for every $A \in \operatorname{Alg} \mathcal{L}$. In particular, if $\delta(I)=0$, then $\delta$ satisfies WJD property if and only if $\delta$ is a derivation.

Corollary 3.4. Let $\mathcal{L}$ be as in Example 1.1. Then $\delta: \operatorname{Alg} \mathcal{L} \rightarrow B(H)$ satisfies WJD property if and only if $\delta$ is a derivation.

Proof. By Theorem 3.2, we only need to show that if $\delta$ satisfies WJD property, then $\delta(I)=0$. Let $n \geq 2$. By [7, Lemma 3.2], we have $\left(P_{n}\right)_{-} \nsucceq P_{n}$. Hence there exist $z_{n} \in P_{n}$ and $g_{n} \in\left(P_{n}\right)_{-}^{\perp}$ such that $g_{n}\left(z_{n}\right)=1$. Also, there exists $y_{n} \in P_{n}$ such that $y_{n}$ and $z_{n}$ are linearly independent. Since $\delta$ satisfies WJD property, we have $\delta(I) A \in \mathcal{A}^{\prime}$ for every $A \in \mathcal{A}$, which implies that there exists some scalar $\lambda_{n}$ such that $\delta(I) x=\lambda_{n} x$ for every $x \in P_{n}$ and $\delta(I)\left(z_{n} \otimes g_{n}\right)\left(y_{n} \otimes g_{n}\right)=$ $\delta(I)\left(y_{n} \otimes g_{n}\right)\left(z_{k} \otimes g_{n}\right)$. That is $\lambda_{n} g_{n}\left(y_{n}\right) z_{n}=\lambda_{n} y_{n}$. The independence of $y_{n}$ and $z_{n}$ gives $\lambda_{n}=0$ and $\delta(I) x=0$ for every $x \in P_{n}$. Since $\vee\left\{P_{n} \in \mathcal{L}: n=2,3, \cdots\right\}=H$, it follows that $\delta(I)=0$. The proof is complete.

Corollary 3.5. Let $\mathcal{L}$ be a subspace lattice on $H$ with $\operatorname{dim} H \geq 2$ such that $\vee\left\{L: L \in \mathcal{P}_{\mathcal{L}}\right\}=H$ or $\wedge\left\{L_{-}: L \in \mathcal{P}_{\mathcal{L}}\right\}=(0)$. If $\mathcal{L}$ has a non-trivial comparable element, then $\delta: \operatorname{Alg} \mathcal{L} \rightarrow B(H)$ satisfies WJD property if and only if $\delta$ is a derivation.

Proof. According to Theorem 3.2, we only need to show that if $\delta$ satisfies WJD property, then $\delta(I)=0$. By [11, Proposition 2.9], we have $(\operatorname{Alg} \mathcal{L})^{\prime}=\mathbb{C} I$. Hence by Theorem 3.2, we have $\delta(I)=\lambda I$ and $\delta(I) A=\mu_{A} I$ for every $A \in \operatorname{Alg} \mathcal{L}$ (where $\lambda, \mu_{A} \in \mathbb{C}$ ). We claim that $\lambda=0$. Suppose that $\lambda \neq 0$, then every operator in $\operatorname{Alg} \mathcal{L}$ is a scalar multiple of the identity $I$. That is, for every $A \in \operatorname{Alg} \mathcal{L}$, the range of $A$ is $H$ or 0 . However, Since $\operatorname{Alg} \mathcal{L}$ contains a rank one operator, it is impossible. Hence $\delta(I)=0$.

By Corollary 3.5, we can easily show the following result.

Corollary 3.6. Let $\mathcal{L}$ be a subspace lattice on $H$ with $\operatorname{dim} H \geq 2$ such that $H_{-} \neq H$ or $(0)_{+} \neq(0)$. Then $\delta: \operatorname{Alg} \mathcal{L} \rightarrow B(H)$ satisfies WJD property if and only if $\delta$ is a derivation.

Remark. It follows from Theorems $2.4,2.5,3.2$ and 3.3 that every linear mapping satisfying WJD property from a $\mathcal{P}$-subspace lattice algebra into $B(X)$ is Jordan derivable at zero point. 
But the converse is not true. For example, let $\mathcal{T}_{2}(\mathbb{C})$ be the algebra of all $2 \times 2$ upper triangular matrices over the complex field $\mathbb{C}$. Define a linear mapping $\delta: \mathcal{T}_{2}(\mathbb{C}) \rightarrow \mathcal{T}_{2}(\mathbb{C})$ according to

$$
\delta\left(\left(\begin{array}{cc}
x_{11} & x_{12} \\
0 & x_{22}
\end{array}\right)\right)=\left(\begin{array}{cc}
x_{11} & x_{11}-x_{22}+x_{12} \\
0 & x_{22}
\end{array}\right),
$$

for all $x_{i j} \in \mathbb{C},(1 \leq i \leq j \leq 2)$. It is easy to show that $\delta$ is a generalized derivation and $\delta(I)=I \in\left(\mathcal{T}_{2}(\mathbb{C})\right)^{\prime}$, that is, $\delta$ is Jordan derivable at zero point. However, it follows from Corollary 3.6 that $\delta$ does not satisfy WJD property.

\section{Derivable mappings at zero point and local generalized derivations}

Let $\mathcal{A}$ be a unital algebra, $\mathcal{M}$ be an $\mathcal{A}$-bimodule and $\mathcal{T}$ be an ideal of $\mathcal{A}$. We say that $\mathcal{T}$ is a left (respectively right) separating set of $\mathcal{M}$ if for every $m$ in $\mathcal{M}, m \mathcal{T}=\{0\}$ implies $m=0$ (respectively $\mathcal{T} m=\{0\}$ implies $m=0$ ). $\mathcal{T}$ is called a separating set of $\mathcal{M}$ if $\mathcal{T}$ is a left separating set and a right separating set of $\mathcal{M}$. The following result is obvious.

Lemma 4.1. Suppose that $\mathcal{L}$ is a subspace lattice on $X$ such that $\vee\left\{L: L \in \mathcal{P}_{\mathcal{L}}\right\}=X$ (respectively $\wedge\left\{L_{-}: L \in \mathcal{P}_{\mathcal{L}}\right\}=(0)$ ). Then the ideal $\mathcal{T}=\operatorname{span}\left\{x \otimes f: x \in E, f \in E_{-}^{\perp}, E \in \mathcal{P}_{\mathcal{L}}\right\}$ of $\operatorname{Alg} \mathcal{L}$ is a left (respectively right) separating set of $B(X)$.

By Lemmas 2.2 and 4.1 , we have the following result.

Theorem 4.2. Let $\mathcal{L}$ be a subspace lattice on $X$ such that $\vee\left\{L: L \in \mathcal{P}_{\mathcal{L}}\right\}=X$ or $\wedge\left\{L_{-}: L \in\right.$ $\left.\mathcal{P}_{\mathcal{L}}\right\}=(0)$ and $\delta$ be a linear mapping from $\operatorname{Alg} \mathcal{L}$ into $B(X)$. Then $\delta$ is derivable at zero point if and only if $\delta$ is a generalized derivation and $\delta(I) \in(\operatorname{Alg} \mathcal{L})^{\prime}$. In particular, if $\delta(I)=0$, then $\delta$ is derivable at zero point if and only if $\delta$ is a derivation.

Proof. We will show that if $\mathcal{L}$ satisfies $\vee\left\{L: L \in \mathcal{P}_{\mathcal{L}}\right\}=X$ and $\delta$ is derivable at zero point, then $\delta$ is a generalized derivation and $\delta(I) \in(\operatorname{Alg} \mathcal{L})^{\prime}$. The proof for $\mathcal{L}$ with $\wedge\left\{L_{-}: L \in \mathcal{P}_{\mathcal{L}}\right\}=(0)$ is similar. By the proof of [10, Lemma 3], we may show that $\delta(A P)=\delta(A) P+A \delta(P)-A \delta(I) P$ and $\delta(I) P=P \delta(I)$, for every $A \in \operatorname{Alg} \mathcal{L}$ and every idempotent $P \in \operatorname{Alg} \mathcal{L}$. With a proof similar to the proof of Claim 1 in Theorem 2.4] we have $\delta(I) \in(\operatorname{Alg} \mathcal{L})^{\prime}$. Now for all $A, B \in \operatorname{Alg} \mathcal{L}$ and $T \in \mathcal{T}$, we have

$$
\delta(A B T)=\delta(A B) T+A B \delta(T)-A B \delta(I) T
$$

and

$$
\begin{aligned}
\delta(A B T)= & \delta(A) B T+A \delta(B T)-A \delta(I) B T \\
= & \delta(A) B T+A \delta(B) T+A B \delta(T) \\
& -A B \delta(I) T-A \delta(I) B T .
\end{aligned}
$$

It follows that $\delta(A B) T=\delta(A) B T+A \delta(B) T-A \delta(I) B T$. Since $\mathcal{T}$ is a left separating set of $B(X)$, we obtain $\delta(A B)=\delta(A) B+A \delta(B) T-A \delta(I) B$ for all $A, B \in \operatorname{Alg} \mathcal{L}$. That is, $\delta$ is a generalized derivation. The proof is complete. 
Recall that a linear mapping $\delta$ from $\mathcal{A}$ into $\mathcal{M}$ is a left (respectively right) multiplier if $\delta(A B)=\delta(A) B($ respectively $\delta(A B)=A \delta(B))$ for all $A, B \in \mathcal{A} ; \delta$ is a local generalized derivation if for every $A \in \mathcal{A}$ there is a generalized derivation $\delta_{A}: \mathcal{A} \rightarrow \mathcal{M}$ (depending on $A$ ) such that $\delta(A)=\delta_{A}(A)$. In the following we give some applications of Lemmas 2.2 and 4.1 , The proofs of the results are similar to the proof of Theorem 4.2. and we leave them to readers.

Theorem 4.3. Suppose that $\mathcal{L}$ is a subspace lattice on $X$ such that $\vee\left\{L: L \in \mathcal{P}_{\mathcal{L}}\right\}=X$ (respectively $\left.\wedge\left\{L_{-}: L \in \mathcal{P}_{\mathcal{L}}\right\}=(0)\right)$ and $\delta$ is a linear mapping from $\operatorname{Alg} \mathcal{L}$ into $B(X)$. Then $\delta$ has the following properties:

(a) if $\delta(A B)=\delta(A) B$ for any $A, B \in \operatorname{Alg} \mathcal{L}$ with $A B=0$, then $\delta$ is a left multiplier (respectively if $\delta(A B)=A \delta(B)$ for any $A, B \in A \lg \mathcal{L}$ with $A B=0$, then $\delta$ is a right multiplier);

(b) if $\delta(A B)=\delta(A) B+\delta(B) A$ for any $A, B \in \operatorname{Alg} \mathcal{L}$ with $A B=0$ and $\delta(I)=0$, then $\delta \equiv 0$ (respectively if $\delta(A B)=A \delta(B)+B \delta(A)$ for any $A, B \in \operatorname{Alg} \mathcal{L}$ with $A B=0$ and $\delta(I)=0$, then $\delta \equiv 0)$

(c) if $\delta\left(A^{2}\right)=2 \delta(A) A$ for all $A \in \operatorname{Alg} \mathcal{L}$, then $\delta \equiv 0$ (respectively if $\delta\left(A^{2}\right)=2 A \delta(A)$ for all $A \in \operatorname{Alg} \mathcal{L}$, then $\delta \equiv 0)$.

Combining Theorem 4.3 (a) and [12, Proposition 1.1], we have

Corollary 4.4. Suppose that $\mathcal{L}$ is a subspace lattice on $X$ such that $\vee\left\{L: L \in \mathcal{P}_{\mathcal{L}}\right\}=X$ and $\wedge\left\{L_{-}: L \in \mathcal{P}_{\mathcal{L}}\right\}=(0)$ and $\delta$ is a linear mapping from $\operatorname{Alg} \mathcal{L}$ into $B(X)$. Then the following are equivalent.

(a) $\delta$ is a generalized derivation.

(b) $\delta$ is a local generalized derivation.

(c) $A \delta(B) C=0$, whenever $A, B, C \in \operatorname{Alg} \mathcal{L}$ such that $A B=B C=0$.

Combining Lemmas 2.2, 4.1 and [13, Theorem 2.8], we also have

Theorem 4.5. Let $\mathcal{L}$ be a subspace lattice on $X$ such that $\vee\left\{L: L \in \mathcal{P}_{\mathcal{L}}\right\}=X$ and $\wedge\left\{L_{-}\right.$: $\left.L \in \mathcal{P}_{\mathcal{L}}\right\}=(0)$. If $h$ is a bijective linear mapping from $\operatorname{Alg} \mathcal{L}$ onto a unital algebra satisfying $h(A) h(B) h(C)=0$ for all $A, B, C \in \operatorname{Alg} \mathcal{L}$ with $A B=B C=0$ and $\delta(I)=I$, then $h$ is an isomorphism.

\section{References}

[1] R. An, J. Hou, Characterizations of Jordan derivations on rings with idempotent - additive maps Jordan derivable at zero, Chin. Ann. Math., 31(2010), 463-474.

[2] M. Bresar, Jordan derivations on semiprime rings, Proc. Amer. Math. Soc., 104(1988), 1003-1006.

[3] M. Chebotar, W. Ke, P. Lee, Maps characterized by action on zero products, Pac. J. Math., 216(2004), 217-228.

[4] Y. Chen, J. Li, Characterizations of Jordan derivations on strongly double triangle subspace lattice algebras, preprint.

[5] F. Gilfeather, R. Moore, Isomorphisms of certain CSL algebras, J. Funct. Anal., 67(1986), 264-291. 
[6] D. Hadwin, J. Li, Local derivations and local automorphisms, J. Math. Anal. Appl., 290(2003), 702-714.

[7] C. Hou, Cohomology of a class of Kadison-Singer algebras, Sci. China Math., 53(2010), 1827-1839.

[8] M. Jiao, J. Hou, Additive maps derivable or Jordan derivable at zero point on nest algebras, Linear Algebra Appl., 432(2010), 2984-2994.

[9] C. Laurie, W. Longstaff, A note on rank-one operators in reflexive algebras, Proc. Amer. Math. Soc., 89(1983), 293-297.

[10] W. Jing, S. Lu, P. Li, Characterisations of derivations on some operator algebras, Bull. Aust. Math. Soc., 66(2002), 227-232.

[11] J. LI, Commutants and double commutants of reflexive algebras, Kyushu J. Math., 50(1996), 171-178.

[12] J. LI, Z. PAN, Annihilator-preserving maps, multipliers and derivations, Linear Algebra Appl., 432(2010), 5-13.

[13] J. Li, Z. PAN, J.Zhou, Isomorphisms and generalized derivations of some algebras, Expo. Math., 28(2010), 365-373.

[14] W. Longstaff, Strongly reflexive lattices, J. London Math. Soc., 11(1975), 491-498.

[15] W. Longstaff, O. Panaia, J-J-subspace lattices and subspace $M$-bases, Stud. Math., 139(2000), 197-212.

[16] F. Lu, Lie derivations of certain CSL algebras, Israel J. Math., 155(2006), 149-156.

[17] F. Lu, The Jordan structure of CSL algebras, Stud. Math., 190(2009), 283-299.

[18] F. Lu, Jordan derivations of reflexive algebras, Integr. Equ. Oper. Theory, 67(2010), 51-56.

[19] F. Lu, B. Liu, Lie derivations of reflexive algebras, Integr. Equ. Oper. Theory, 64(2009), 261-271.

[20] L. Wang, W. Yuan, A new class of Kadison-Singer algebras, Expo. Math., to appear.

[21] S. Zнао, J. Zhu, Jordan all-derivable points in the algebra of all upper triangular matrices, Linear Algebra Appl., 433(2010), 1922-1938. 